\title{
Italique
}

Poésie italienne de la Renaissance

I| 1998

Varia

\section{Il vestito sconveniente. Abiti et armature nella Secchia Rapita}

Silvia Longhi

\section{OpenEdition}

Journals

\section{Edizione digitale}

URL: http://journals.openedition.org/italique/96

DOI: 10.4000/italique.96

ISSN: 1663-4438

\section{Editore}

Librairie Droz

\section{Edizione cartacea}

Data di pubblicazione: 1 giugno 1998

Paginazione: 103-126

ISBN: 2-600-00239-1

ISSN: 1423-3983

Notizia bibliografica digitale

Silvia Longhi, « II vestito sconveniente. Abiti et armature nella Secchia Rapita », Italique [Online], I | 1998, online dal 19 novembre 2009, consultato il 20 avril 2019. URL : http://journals.openedition.org/ italique/96 ; DOI : 10.4000/italique.96 
SILVIA LONGHI

IL VESTITO SCONVENIENTE.

ABITI E ARMATURE NELLA

SECCHIA RAPITA 


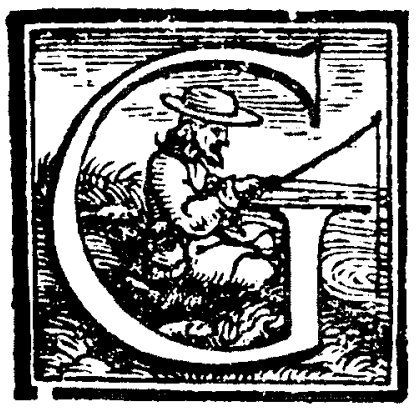

LI «accidenti strani» che il Tassoni promette ai suoi lettori nell'esordio della Secchia rapita si manifestano subito con un rovesciamento delle ordinate abitudini quotidiane.' L'incursione mattutina dei Bolognesi sveglia di botto gli abitanti di Modena, e li obbliga a una preparazione veloce e scombinata (1 11):

chi si mise una scarpa e una pianella, e chi una gamba sola avea calzata, chi si vestì a rovescio la gonella, chi cambiò la camicia con l'amata; fu chi prese per targa una padella $e$ un secchio in testa in cambio di celata, e chi con un roncone e la corazza corse bravando e minacciando in piazza.

I Modenesi si vestono: con indumenti male assortiti (1), a metà (2), con abiti indossati al contrario (3), con abiti scambiati tra uomo e donna (4), con armi improprie $(5,6,7)$. Il delizioso tocco del usecchio" trasformato in elmo introduce un duplicato domestico, se possibile ancor più dimesso, della "vil secchia di legno" che muove l'azione. L'ottava è il primo esempio di un impiego anomalo delle vesti che si riprodurrà per tutto il corso del poema: vesti e armature bizzarre, mal combinate, inadatte alla persona che le indossa, falsificate, usurpate: in una parola, sconvenienti.

Le armature burlesche

La rassegna dell'esercito modenese, nel canto III, annovera anche milizie povere e scalcinate, se non selvatiche. Montanari e cacciatori, venuti dai borghi dell'Appennino (57, 5-8):

con archi e spiedi porcherecci in mano, spiegando in campo bianco una padella; trecento fur che quelle vie ronchiose con le piante premean dure e callose; 
porcai, taglialegna, contadini, e ancora cacciatori (58, 1-8):

Seguiva di Monforte e di Montese,

Montespecchio e Trentin poscia l'insegna:

Gualtier figliuol di Paganel Cortese

l'avea dipinta d'una porca pregna;

fur quattrocento, e parte al tergo appese

accette avean da far nel bosco legna,

parte forconi in spalla, e parte mazze

e pelli d'orsi in cambio di corazze.

L'epopea di questa "gente indomita e silvestra" $(62,2)$, di questi "inculti abitator de l'Apennino" $(63,4)$, culmina nell'ottava 64:

Tutti a piedi venian con gli stivali, armati di balestre a martinelle che facevano colpi aspri e mortali

e passavano $i$ giacchi e le rotelle: pelliccioni di lupi e di cinghiali eran le vesti lor pompose e belle; spadacce al flanco aveano e stocchi antichi, e cappelline in testa e pappafichi.

Commenta la nota del Salviani: "Niuna cosa vien istimata piu abile a muovere il riso che gli abiti contrafatti; e però il poeta arma questi popoli montagnuoli così alla scapigliata".

L'espediente è antico. Più di una volta il Tassoni sente il bisogno di agganciare la genealogia della Secchia direttamente alla Batracomiomachia: dal poemetto pseudo-omerico prende avvio il discorso di Giove, quando parlando nel Concilio degli dè ricostruisce gli antefatti classici delle battaglie che si preparano tra $i$ moderni guerrieri bolognesi e modenesi: "ei cominciò dal di che fu ripieno / di topi il mondo e di ranocchi spenti” (II 43, 5-6); da li deve riprendere il canto la nuova Musa eroicomica: "Musa, tu che cantasti i fatti egregi / del re de' topi e de le rane antiche, / sì che ne sono ancor fioriti i fregi / là per le piagge d'Elicona apriche, / tu dimmi) (V 23, 1-5); e non per caso ogni tanto si inciampa in un paragone con il topo, la rana o il ranocchio." Uno dei capitani modenesi, che evidentemente se ne intendeva, "per insegna avea una rana I armata con la spada e la rotella» (III 22, 5-6). Nella Batracomiomachia l'abbassamento dell'epica si realizza con la parodia di molti temi e motivi 
fissi, e tra questi ba un grande rilievo la scena della vestizione dei combattenti (che tuttavia si vestono partendo dal basso verso l'alto, cioè secondo l'ordine consueto). ${ }^{+}$I topi si servono di fave come schinieri, pelli di donnola come corazze, cuori di lucerne come scudi, aghi come lance, scorze di ceci come elmi (123-31). Le rane usano foglie di malva come calzari, bietole verdi come corazze, foglie di cavolo come scudi, giunchi come lance, gusci di chiocciole come elmi (160-65). Le armi sono dunque di origine vegetale (e in certi casi si tratta di vegetali commestibili: cibi poveri), di origine animale, o provengono dall'arredo domestico.

L'altro modello classico cui Giove allude nello stesso discorso inaugurale è la Storia vera di Luciano: "e narrò le battaglie ad una ad una / che ne' campi seguir poi de la luna" (II 43, 7-8). Presso Luciano, gli abitanti del Sole e quelli della Luna combattono con armi vegetali: adoperano fave come elmi, gusci di lupino come corazze, funghi come scudi, gambi di asparagi come lance, ravanelli avvelenati con malva come proiettili (I 1416). Tutte le risorse del regno animale sono invece riservate ai corpi stessi dei combattenti-mostri inventati da Luciano, straordinario maestro di zoologia fantastica. ${ }^{5}$

La specializzazione vegetale è propria anche dell'armamento degli insetti nella Moscheis del Folengo (ricordiamo che un energico elogio di Merlin Cocai è nel canto VIII, 24-25 della Secchia). Nella rassegna dell'esercito delle mosche, Sanguileone porta un'armatura fatta di scorza di cece nero, mezzo guscio di fagiolo come scudo, il pelo di una scrofa come lancia, metà granello di miglio come celata, un guscio di fava come panciera (I 367-72). ${ }^{6}$

A riscontro dei suoi modelli, il Tassoni impiega uno scarto diverso, ma dal punto di vista concettuale simmetrico a quelli considerati: si tratta sempre di scendere di un gradino. I protagonisti umani della Secchia portano, in servizio del comico, armi degradate: inalberano insegne ridicole, e usano strumenti impropri, adatti all'agricoltura o alla caccia, attività meno nobili della guerra. Il duello finale del poema, tra i burleschi eroi Sprangone e Lemizzone (uno enorme e pesante; l'altro più piccolo e agile), non è a colpi di spada, ma di "ronca" e "rampicone» (XII 49, 4; 51, 3; 53, le 5, ecc.). La ronca è prima un attrezzo agricolo che un'arma: un'asta di legno a cui è fissata una grossa lama ricurva, a doppio taglio nella varietà specifica "ronca bolognese» (49, 4): è insomma la falce dei contadini. Il rampicone è un uncino di ferro fissato a un'impugnatura, propriamente un attrezzo marinaresco, che serve per abbordare imbarcazioni o per cacciare animali marini: qui arpiona alla cintola Sprangone, un bestione terrestre che caduto in 
acqua annega (per l'impaccio dell'armatura e delle brache slacciate, o forse perché non sa nuotare).

\title{
La guerriera travestita
}

Fra le milizie che escono da Modena per l'impresa di Bologna, ci sono anche "cento donzelle in abito guerriero / col fianco e 'l petto di corazza armato, / e l'aste in mano e le celate in testa" (I 15, 5-7). Altre cinquanta donne scendono poi dai monti "con gli archi in mano / avezze al bosco a saettar le fiere / e a colpir da vicino e da lontano, / succinte in gonna e faretrate arciere / (...) / e la chioma bizarra e ad arte incolta / ondeggiando su' 'l tergo iva disciolta" (III 50, 2-8). L'aspetto dei due gruppi di guerriere in parte è improntato alla tradizionale fisionomia del cavaliere medievale, in parte contiene elementi alieni, desunti dal modello classico delle Amazzoni, ovvero da quello delle seguaci di Artemide cacciatrice. La prima delle cento è Renoppia, il cui nome conserva una traccia proprio dei nomi delle sorelle Amazzoni Ippolita, Antiope e Melanippe, protagoniste di vicende legate a Ercole e a Teseo (I 16):

\author{
Venìn guidate da Renoppia bella \\ cacciatrice ed arciera a l'armi avezza; \\ Renoppia di Gherardo era sorella, \\ pari a lui di valor, di gentilezza, \\ ma non avea l'Italia altra donzella \\ pari di grazia a lei né di bellezza, \\ e parea co' virili atti e sembianti \\ rapir i cori e spaventar gl'amanti.
}

La "virile» Renoppia, donzella mascolina che attrae e insieme terrorizza $i$ suoi innamorati, è un'immagine mista e ambivalente.

Com'è noto, il tema della donna guerriera, vestita con una normale armatura maschile, ha una salda fortuna nella narrativa epicocavalleresca:" l'identità femminile si manifesta sempre quando, al levar dell'elmo, si scioglie la treccia bionda. In questo modo, presso il Boiardo, Ruggiero vede per la prima volta la faccia di Bradamante, e se ne innamora: "Nel trar de l'elmo si sciolse la treccia, / Che era de color d'oro allo splendore. / Avea il suo viso una delicateccia / Mescolata di ardire e de vigore" (Orlando innamorato III V 41, 1-4). L'equivoco avvolge Bradamante quanto più ci si avvicina allinterruzione del poema: ferita, $e$ 
ancora armata, arriva per sorte alla dimora di un eremita, e si presenta con una formula menzognera: "Io sono un cavallier, - disse la dama - I Ch'ier me smaritti in questa selva oscura» (III VIII 55, 1-2). L'eremita, che vorrebbe medicare il presunto cavaliere ferito - levato di mezzo l'elmo alla vista della treccia si sgomenta "Vide la trezza e fo tutto smarito" $(60,4)$; poi si risolve a tagliare $i$ capelli: "Benché convenne le chiome tagliare / Per la ferita, che era grande e strana: / Le chiome li tagliò come a garzone" $(61,5-7)$. Ora la fisionomia maschile è perfetta anche senza l'elmo: mentre dorme a capo scoperto sulla riva di un fiume, Bradamante fa innamorare di sé Fiordispina, che la scambia per un bel cavaliere (62-66). E il Boiardo abbandona i suoi lettori proprio nel mezzo di «questo vano amore» (III IX 26,5).

I giochi dell'elmo e delle chiome ricominciano nell'Orlando furioso, ${ }^{9}$ dove Bradamante entra in scena come un uomo: "Ecco pel bosco un cavallier venire, I il cui sembiante è d'uom gagliardo e fiero: / candido come nieve è il suo vestire, I un bianco pennoncello ha per cimiero" (I 60, 1-4). Solo al canto XXV l'Ariosto si decide a riprendere la vicenda del vano amore di Fiordispina, all'interno del racconto che ne fa il gemello di Bradamante, Ricciardetto, a lei somigliantissimo e perciò destinato a porre rimedio, con una sostituzione di persona, all'impossibilità di quell'amore. L'esordio del racconto di Ricciardetto è tutto dominato dall'impressione prepotente di quel taglio di capelli della sorella, che viene detto e ridetto: scorciati i "lunghi crini", già "segno (...) di differenzia", trionfano le "viril fattezze" della guerriera (23-28). Bradamante (che nell'Orlando innamorato si era machiata di una colpevole reticenza in proposito) disinganna però subito Fiordispina, dichiarandosi "donzella», e confrontandosi al modello della guerriera di tradizione classica, "che gloria, qual già Ippolita e Camilla, I cerca ne l'armes (32, 1-2). Non mi fermerò sull'episodio degli amori di Fiordispina con Bradamante-Ricciardetto, che è già stato ottimamente studiato. ${ }^{10}$ All'altezza del canto XXXI, quando Bradamante arriva alla rocca di Tristano, combatte, vince e si guadagna l'ospitalità, i capelli sono ormai ricresciuti:

La donna, cominciando a disarmarsi,

s'avea lo scudo e dipoi l'elmo tratto;

quando una cuffia d'oro, in che celarsi

soleano i capei lunghi e star di piatto,

uscì con l'elmo; onde caderon sparsi

giù per le spalle, e la scopriro a un tratto

e la feron conoscer per donzella,

non men che fiera in arme, in viso bella.

(XXXII, 79, 1-8) 
Già son cresciute e fatte lunghe in modo

le belle chiome che tagliolle il frate,

che dietro al capo ne può fare un nodo,

ben che non sian come son prima state.

(XXXII, 81, 1-4)

Ma con un'argomentazione inattesa, mossa da un fine di somma cortesia (Bradamante non vuole che, secondo la legge del luogo, sia allontanata l'altra donna presente, giudicata meno bella di lei), la guerriera rinnega l'evidenza di quel segno di riconoscimento esteriore dei capelli, finora universalmente valido: "Ben son degli altri ancor, channo le chiome I lunghe, com'io, né donne son per queston; e dichiara di voler essere considerata non una donna, ma un cavaliere, dato che in qualità di cavaliere ha combattuto e meritato asilo nella rocca: "perché dunque volete darmi nome / di donna, se di maschio è ogni mio gesto?» (103, 1-2 e 5-6). Qui non è solo questione di vesti, né di sembiante o fattezze, ma di gesti e comportamenti; e la bilancia dell'ambigua identità di Bradamante inclina dalla parte maschile.

La prima apparizione in scena della Clorinda tassiana ${ }^{11}$ sembra ricalcare il primo ingresso di Bradamante nel Furioso, citato sopra: "ecco un guerriero / (ché tal parea) d'alta sembianza e degna» (Gerusalemme liberata II 38, 1-2); in altra occasione si dice che vestiva, anche lei, di bianco: "Bianche via più che neve in giogo alpino / avea le sopraveste" (VI 26, 56). A volte Clorinda tiene la visiera sollevata, concedendo una visione almeno parziale del bel volto (I 47, 2; VI 26, 6-7). Anche per lei comunque vale il solito effetto di subitanea, folgorante rivelazione che si produce al levar dell'elmo, qui per un colpo di lancia alla visiera durante uno scontro: "ché, rotti i lacci a l'elmo suo, d'un salto / (mirabil colpo!) ei le balzò di testa; / e le chiome dorate al vento sparse, / giovane donna in mezzo 'l campo apparse" (III 21, 5-8). La natura ancipite, e anomala nella doppiezza, di Clorinda (pagana, e poi cristiana; guerriero di "maschio valore», molto più che donna allenata al combattimento) è stata già interpretata in modo convincente quale conseguenza necessaria della storia del personaggio, posto sotto il segno dell'anomalia fin dalle circostanze, singolarmente distorte, della sua nascita e educazione.12 Dirò qualcosa invece di un travestimento vero e proprio, che indirettamente implica Clorinda, o meglio la sua armatura. Erminia, che è tanto fermminea quanto Clorinda è mascolina, mentre passa il tempo struggendosi per l'amato Tancredi che immagina ferito, vede nelle stanze dell'amica lillustre panoplia appesa, e la contempla con invidia: "sospese di Clorinda 
in alto mira / l'arme e le sopraveste: allor sospira” (VI 81, 7-8); il suo voto impossibile è di avere un corpo diverso, abbastanza forte da poter "la gonna e'l velo / cangiar ne la corazza e ne l'elmetto" (83, 3-4). Detto fatto, Erminia si traveste da Clorinda, se non altro per uscire impunemente dalla città: "Io guerreggiar non già, vuo' solamente / far con questarmi un ingegnoso inganno: / finger mi vuo' Clorinda; e ricoperta / sotto l'imagin sua, d'uscir son certa" $(87,5-8)$. Ė una vestizione innaturale, perché la durezza e il peso dell'armatura disconvengono al corpo sottile e morbido contenuto all'interno: "Co 'l durissimo acciar preme ed offende / il delicato collo e l'aurea chioma, I e la tenera man lo scudo prende, I pur troppo grave e insopportabil soma" (92, 1-4). La sconvenienza è misurata dal paragone con il travestimento femminile (dunque opposto e simmetrico) di Ercole, ridotto in vesti muliebri da Iole: "Gode Amor ch"è presente, e tra sé ride, I come allor già ch'avolse in gonna Alcide" (92, 7-8). A rinforzo, l'ottava 3 del canto XVI presenterà il duplice scambio di abiti e oggetti tra Ercole e Iole: scambio risibile e vituperoso, che si rivela come il parametro antico su cui commisurare ogni infrazione al decoro e alla proprietà dell'abito:

\author{
Mirasi qui fra le meonie ancelle \\ favoleggiar con la conocchia Alcide. \\ Se l'inferno espugnò, resse le stelle, \\ or torce il fuso; Amor se 'l guarda, e ride. \\ Mirasi Iole con la destra imbelle \\ per ischerno trattar l'armi omicide; \\ e indosso ha il cuoio del leon, che sembra \\ ruvido troppo a sì tenere membra.
}

Torniamo alla Secchia. Di contro a tanta biondezza sparsa nella rassegna delle eroine rinascimentali, spiccano come uno scarto dalla norma i capelli scuri di Renoppia: "Bruni gli occhi e i capegli, e rilucenti" (I 17, 1). La curiosità invita a cercare ritratti di Renoppia lungo le molte edizioni illustrate della Secchia, ricorrendo ai lavori di Pietro Puliatti sull'iconografia tassoniana. ${ }^{13}$ In effetti, tra gli episodi figurati abbastanza spesso c'è quello cosiddetto dell" "aedo Scarpinello", corrispondente a VIII 45-75: in una pausa del combattimento, Renoppia e alcune compagne, occupate nel ricamo, ricevono la visita degli ambasciatori bolognesi, e con loro ascoltano il canto dellimprovvisatore cieco Scarpinello, chiamato a intrattenere gli ospiti. Renoppia interromperà sdegnata la prima storia, lasciva, degli amori di Endimione con la Luna; e poi anche la seconda storia, apparentemente seria, dell'assalto di Tarquinio alla casta Lucrezia. 


\section{Silvia LongHI}

Le ragioni di questa duplice interruzione sono già state brillantemente chiarite da uno studio di Ottavio Besomi, ${ }^{14}$ con cui tra poco dovrò dialogare. Ma intanto, lillustrazione su cui conviene fermarsi è una xilografia anonima dell'edizione Soliani minor del 1744 (la seconda, e più modesta, delle due pubblicate a Modena nello stesso anno da Bartolomeo Soliani). ${ }^{15}$ Renoppia è nel suo padiglione, seduta davanti al telaio da ricamo, con la destra alzata nell'atto di lanciare la pantofola contro Scarpinello. $\dot{E}$ raffigurata in abiti femminili (come di norma nelle rappresentazioni di questo episodio); e però in alto, sopra la sua testa, è appesa al muro l'armatura, vuota come un guscio vuoto. Così è significata, con originale ed efficacissima sintesi, la doppia natura della guerriera. Un'altra invenzione molto attraente di questa xilografia è una finestra del fondo spalancata sul buio della notte, che mostra (sopra un lontano cavaliere a cavallo) la falce della luna: la finestra cioè "contiene" una delle due storie di Scarpinello.

Ora qualche osservazione sul testo. L'argomento del canto VIII, "son da Bologna ambasciator mandati / che di Renoppia fra i ricami e l'armi I del cieco Scarpinello odono i carmi" (6-8), salda felicemente in unità anagrammatica $i$ RICAMI e le ARMI in cui la guerriera esercita uguale maestria, e ci aggiunge una più enigmatica solidarietà fonica con $i$ CARMI del cieco. Besomi ha mostrato la forza provocatoria del primo carme, sugli amori della Luna, che offende Renoppia per la sua morale scostumata: la Luna, che è anche Artemide-Diana, si pente dei suoi anni casti, sprecati a cacciare nei boschi (il suo discorso paradossale è alle ottave 60-62). Renoppia si indigna - aggiungiamo - non solo in quanto casta, ma perché vede messo in discussione uno dei suoi modelli mitologici. All'inizio della scena ci viene detto, di Renoppia e compagne, che «Stavano a' lor ricami intente armate / imitando Minerva in ogni parte» (45, 5-6): ciò̀ imitavano Pallade-Minerva in entrambe le sue specialità, combattere e tessere (per questultima testimonia Ovidio, Metamorfosi VI 1-145). Il Tassoni non dice, ma è implicito, che queste guerriere imitano anche Artemide-Diana, da sempre considerata la protettrice delle Amazzoni (che come la dea sono arciere e cacciatrici, $e$ stanno alla larga dagli uomini). Quindi si capisce che Renoppia non voglia piu ascoltare la sua dea tutelare mentre si smentisce, imponendo alle donne una nuova "legge» amorosa, contraria a quella, severissima, che soleva imporre alle sue seguaci (la storia di Callisto insegna: Ovidio, Metamorfosi II 401-65). La guerriera suggerisce allora a Scarpinello due argomenti alternativi: "E se vuoi ch"io t'ascolti e che il tuo canto I ritrovi adito piu per queste porte, / cantami di Zenobia il pregio e ' $l$ 
vanto / o di Lucrezia l'onorata morte" $(64,1-4)$; il cantore sceglie il secondo argomento, che tratta però con dovizia di particolari comici, $e$ che è obbligato a interrompere al punto culminante dello stupro. Besomi, ripercorrendo una ricchissima serie di testi sulla castità esemplare di Lucrezia, individua una controcorrente dubitativa o addirittura polemica (Sant'Agostino, poi Bèze e Estienne, e più da presso il Marino), che parla di un possibile consenso di Lucrezia al piacere amoroso, autopunito subito col suicidio; o ancor peggio (Adone XI 53-54), di una morte che punisce lo sciocco errore della castità precedente. Donde la giusta conclusione dello studioso: Renoppia interrompe un'altra volta Scarpinello, perché non vuol correre il rischio di ascoltare Lucrezia dire le stesse cose che aveva detto la Luna.

Petrarca può darci un aiuto per fare ancora un passo. "' Zenobia è un personaggio del Triumphus Fame (II 107-17), e marcia precisamente nella fila formata dalle Amazzoni e altre donne guerriere: la precedono le quattro sorelle Amazzoni Antiope, Orizia, Ippolita e Menalippe, poi la regina Tamiri, l'altra Amazzone Pentesilea, la latina Camilla, Semiramide e Cleopatra. La regina orientale Zenobia è insigne per castità (108), bellezza giovanile (109-11), e coraggio in guerra, dimostrato attaccando i Romani sprezzatori del pericolo: "Nel cor femineo fu si gran fermezza / che col bel viso e coll'armata coma / fece temer chi per natura sprezza" (112-14). Il dettaglio dell" "armata coma" ci è familiare. Nell'insieme, tutto questo corteo di guerriere è genealogia di Renoppia, e lei lo sa: se Diana si è rivelata un modello fallace, si celebri dunque Zenobia, che le sembra ancora affidabile. Anche la proposta di celebrare Lucrezia si spiega bene con un riscontro petrarchesco: Lucrezia ha posto naturalmente nel Triumphus Pudicitie, anzi è la prima, nel corteo delle donne caste (132). Non solo, la vediamo compiere con le altre un gesto sorprendente: "Queste gli strali / avean spezato, e la pharetra a lato / a quel protervo, $e$ spennachiato l'ali» (133-35). Agli occhi di Renoppia, il pregio di Lucrezia è certamente aumentato dal modo tenuto in questa vendetta su Amore: aver fatto a pezzi le frecce e la faretra di Amore, armi impiegate per uno scopo perverso, secondo la discrezione di lei, casta arciera. Riassumendo questa opzione in termini di stile: per ragioni personali, ma ben documentate culturalmente, Renoppia non vuole poesia lirica e amorosa (niente Cariteo, con $i$ suoi incanti lunari e il suo Endimione); vuole bensi poesia trionfale, che sintoni al suo statuto di guerriera, e che onori valori suoi: la Castità, o la Fama, o entrambe. 


\section{Gli dèi travestiti}

Gli dèi convergono nel celeste Concilio con abiti, si sa, più moderni che greci, nella mimesi parodica di uniadunanza solenne della corte papale secentesca. Fermiamo lo sguardo solo su Apollo, in manto rosso e copricapo di velluto, con al collo la decorazione del toson d'oro spagnalo (II 30, 5-6): proprio lui, abituato in tempi antichi ai travestimenti più umili, da pastore o da contadino (Ovidio, Metamorfosi II 679-82, vI 122-24). E Pallade, in una curiosa mise mescidata: «succinta a mezza gamba, in un raccolto I abito mezzo greco e mezzo ispano" $(31,3-4)$, che fa pensare agli abbigliamenti "a metà" di I 11; mentre l'acconciatura "mezzo e mezzo" ("parte il crine annodato e parte sciolto / portava" 31, 5-6) è tutt'altro che una moda esotica o un segno di trascuratezza. La schiera delle donne guerriere di Triumphus Fame II, evocata piu sopra, ci consente di recuperare il senso vero: il modello è Semiramide, "la magnanima reina, I china treccia ravolta e l'altra sparsa / corse a la babilonica rapinas (103105), troppo impaziente di combattere per terminare di pettinarsi. Nella folla dei dignitari poi c'è Ercole, non del tutto guarito dalla pazzia, ciò̀ matto a metà; e travestito da guardia svizzera del papa: con l'uniforme bicolore, a strisce gialle e blu, cioè "mezzo e mezzo" (38, 7-8; 39, 1-8).

Più avanti nello stesso canto II, scende sulla terra in aiuto ai Modenesi un terzetto divino, formato da Venere e dai suoi due spasimanti Bacco e Marte. Si fermano per la notte in un'osteria, ma la dea, con un calcolo che si rivelerà improvvido, ci arriva in abiti maschili: "La diva, per non dar di sé sospetto, ' presa la forma avea d'un giovinetto" (58, 7-8). L'abito di Citerea-garzone è bianco e rosso: seta candida, nella foggia a tagli, che lasciano emergere sboffi di seta vermiglia $(59,1-2)$. L'oste, anziché pensar male come avrebbe fatto alla vista di una donna, pensa malissimo, "quando corcarsi in terzo egli comprese / l'amoroso garzon fra tanti letti” (60,3-4). Gli dè sgomberano rapidamente; giunti a Modena, vengono scambiati per attori della commedia dell'arte: "e molti li tenean per recitanti / venuti a preparar comedie inanti" (61, 7-8). Citerea, vestita da ragazzo, viene presa per un ragazzo autentico, "ammaestrato" a travestirsi da donna per impersonare, secondo la consuetudine, ruoli femminili $(62,3-4)$. La ragione di questo acume interpretativo sta nell'abitudine locale alle mascherate, attestata dallo stesso Bacco, che chiama Modena "La città ch'ognor vive in feste e canti / fra maschere e tornei" (II 50, 1-2). '" L'usanza è comprovata all'ultimo canto: per festeggiare il legato papale "in maschera s'andò mattina e sera" (XII 21, 8), e al momento del congedo, insieme a una montagna di delizie mangerecce, la città gli donò «una cassa di maschere bellissime» $(38,2)$. 
Venere si traveste un'altra volta nel poema, quando assume il "bel sembiante" della contessa di Caserta, sorellastra di Manfredi e sua amante incestuosa, per indurre il principe alla guerra (X 27-37). In questo caso si tratta di uno stratagemma convenzionale, a cui gli dèi dell'epica sono adusati fin dalle origini, perportare aiuto a questa o quella parte rivale. Segue lo stesso procedimento Marte, "che presa la sembianza fiera / di Scalandrone da Bismanta avea, / bandito e capitan di gente rea" (IV 17, 6-8: manterrà tale sembianza fiera fino all'ottava 66). Scalandrone da Bismanta, per parte sua (avvertono $i$ commentatori, ${ }^{18}$ sulla scorta delle ricerche storiche di Venceslao Santi)," non è affatto Scalandrone, bensì il travestimento giocoso del marchese Ernesto Bevilacqua, questo persona vera, e nella vita reale nemico del Tassoni. Allora si pensa a tutto quello che per noi, lettori moderni, s'è perduto del gusto della Secchia: a come invece dovevano divertirsi (o irritarsi) $i$ contemporanei, riconoscendo in continuazione amici e nemici reali, sotto le vesti dei personaggi del poema. Quelle rassegne di armati, quelle serie estenuanti di ottave onomastiche che a noi paiono atone, e senza scintilla, come una parodia di cui si sia perduto l'originale, dovevano brillare al loro tempo, e pungere. Il nuovo commento organico che si prepara, per opera di Besomi e dei suoi collaboratori, restituirà, se non tutto il piacere del testo, almeno la ragionata cognizione di quel piacere.

La moglie del Conte

La logica delle falsificazioni morde a fondo anche nel canto $X$, aggiunto nella redazione finale del poema. L'episodio del Conte di Culagna, avvelenatore avvelenato, si svolge tutto all'insegna dei capovolgimenti e delle sostituzioni fallaci. Il medico Sigonio dà al Conte, che gli aveva chiesto veleno, "una presa d'antimonio"; il Conte si prende l'antimonio "per tossico", e poi sparge questo antimonio da lui creduto tossico sulla minestra della moglie, cercando di far credere a lei che sia pepe (45-48). Alla moglie basta un gesto, il semplice scambio dei due piatti ("e la minestra sua gli cambia in fretta» 49,4): ancora una sostituzione, ancora una cosa per l'altra.

Dopo l'esito della beffa che tutti conoscono, la moglie del Conte pensa bene di travestirsi, per raggiungere di nascosto al campo l'innamorato Titta. Si veste da uomo: "aveva in fretta il suo destrier sellato: / $e$ in abito virile e sconosciuta / con un cappello in testa da soldato / tacitamente già s'era partita" (58, 4-7). Quell'avverbio, "tacitamente», è una suprema finezza 
parodica, che rimanda a Orlando, alla partenza notturna e solitaria di Orlando, irriconoscibile perché travestito con sopravveste scura: "Tacitamente è della terra uscito" presso il Boiardo (Orlando innamorato I II 28, 6); "Da mezza notte tacito si parte» presso l'Ariosto (Orlando furioso VIII 86, 1). La donna si annuncia come "paggio", e difatti sembra un "giovinetto"; Titta la riconosce, ma tutti gli altri no, e pensano male vedendo le loro effusioni: "Parve l'atto ad alcun poco decente / che l'ebbero per maschio a prima mira; / né distinguendo ben dal pesco il fico, I dicevano di lui quel chio non dicon $(60,5-8) . \dot{E}$ lampante la simmetria con il travestimento da "giovinetto" di Citerea, che scandalizzava l'oste vittima di un identico equivoco. Colpisce il fatto che $i$ due episodi paralleli cadano nelle stesse ottave dei rispettivi canti: 58-60 del canto II, Citerea; 58-60 del canto X, la moglie del Conte. Siccome è molto improbabile che questa identità di posizione sia casuale, dovremo dedurne che intende segnalare qualcosa? ci sarà un significato riposto, un'allusione a qualcuno, magari un segreto, indicato da quell'espressione di reticenza "quel ch"io non dico"? Giro volentieri la domanda a chi ne sa più di me.

Ma non è finita. Il Conte si mette alla ricerca della moglie e del cavallo perduti. Titta allora fa tingere il cavallo "con un color di sandali alterato, I e di leardo il fa sauro bruciato" (65, 7-8): travestito così, da grigio qual era (col mantello misto di peli bianchi e neri), in color cannella scuro, il buon destriero non viene riconosciuto dal suo padrone. Poi tocca alla moglie del Conte. Il primo travestimento, da donna a uomo, sera compiuto con un cambio di abiti; quest altro - restando ferme le fattezze femminili - si attua con un cambio di pelle: da candida a bruna (di un colore fulvo carico), grazie al succo del mallo di noce $(68,1-8)$ :

\footnotetext{
Con lei s'accorda, e trova acqua stillata

da scorza fresca di matura noce,

e 'I bel collo e la faccia dilicata

de la donna e le man bagna veloce;

si disperde il candore, e sembra nata

in Mauritania, là dove il sol cuoce,

d'un leonato scuro ella diviene,

ma grazia in quel colore anco ritiene.
}

Completa la trasformazione "un leggiadro abito moro", cioè un costume moresco da schiava $(70,1-4)$, e la metamorfosi è così perfetta che comporta anche il cambio del nome: "Bella mora", la saluta infatti il povero Conte 
$(71,3)$. Ma di quella pelle mutata e falsa (travestimento più intimo non potrebbe darsi), il Tassoni parla, con paragone di mirabile coerenza, come di una stoffa tinta $(69,1-6)$ :

$$
\begin{aligned}
& \text { Come panno di grana in bigio tinto } \\
& \text { ritiene ancor de la beltà primiera, } \\
& \text { e nel morto color d'un nero estinto } \\
& \text { purpureggiar si vede in vista altera; } \\
& \text { così di quella faccia il color finto } \\
& \text { ritiene ancor de la bellezza vera. }
\end{aligned}
$$

La stoffa di porpora tinta di grigio lascia balenare, nell'opacità del nero smorto, riflessi di rosso: è un drappo cangiante, il suo preferito.

Il bianco, il nero e il rosso

Il canto IX è molto misterioso, pieno di prodigi e di complicati rituali cavallereschi. Tra gli enigmi, c'è quello dei paggi sulla strana nave-isola: al di là del significato, che sfugge, risalta la simmetria con le metamorfosi appena considerate della moglie del Conte. Dal padiglione escono "cento paggi in bianca vesta" $(13,7)$, ma neri di pelle: "Bruni i fanciulli avean le mani e 'l viso, I e parean tutti in Etiopia nati" (14, 1-2). Li segue "il cavalier de la ventura", che ha armatura, sopravveste e cimiero bianchi, ma monta un cavallo nero $(16,1-8)$. Tutto il contrario avviene poco piu avanti. Il cavaliere dell'isola si ripresenta vestito di nero, "in veste bruna, / bruno il cimiero e bruno il guarnimento", ma montato su un cavallo bianco (57, 6-8). I paggi da mori sono diventati bianchi come angeli, "re come $i$ visi ancor cangiar livrea", perché ora sono vestiti di nero $(58,1-5$ : l'ottava 58 è decisamente legata alle trasformazioni). Nell'ordine delle due serie di ottave interessate $(13+14+16 ; 57+58)$, il rovesciamento descrive una figura di chiasmo (cioè un ulteriore scambio, di posizione nel testo): la combinazione di paggi neri vestiti di bianco + cavaliere vestito di bianco su cavallo nero, si ribalta nella combinazione di cavaliere vestito di nero su cavallo bianco + paggi bianchi vestiti di nero. ${ }^{20}$

Il canto XI mette in scena il grande duello tra Titta e il Conte di Culagna. Titta compare in campo armato di tutto punto e abbigliato completamente di nero: "armato già dal capo al piede / con armi e piume nere e neri ammanti» (30, 5-6); il Conte, all'opposto, indossa solo una metà dell'armatura "con sopravesta bianca e rilucente» $(32,4)$, e finisce di armarsi 
sul campo, dove i suoi molti accompagnatori gli passano, un pezzo alla volta, elmo, spada, fodero, scudo, lancia, manopole, speroni, cuffia (33-34; l'ordine non è dei più economici). Tale laboriosa vestizione viene disfatta quasi immediatamente, dopo il primo colpo di lancia dell'avversario (che coglie il Conte al collo, là dove l'elmo è fissato alla corazza dalla goletta), con l'aiuto di una schiera ancor più numerosa di persone: "Il portano a la tenda, e sopra un letto / gli cominciano l'armi e i panni a sciorre" (38, 1-2): l'operazione si svolge «in fretta», e viene nominato solo l'elmo. Si noti che il testamento del Conte, con cui lascia le armi, una per una, a degni eredi (lancia, scudo, spada, corsaletto, guanti, bracciali, elmo, cimiero) non trova luogo a questo punto, perché era già stato fatto: il testamento precedeva il duello, invece di seguirlo (14-16). Il Conte si è creduto ferito, e vicino a morte, in virtù di una brillante e malefica invenzione del Tassoni: "Caduta la visiera il Conte mira, / e vede rosseggiar la sopravesta, / e - Oimé son morto, - e' grida; e'l guardo gira / a gli scudieri suoi con faccia mesta" (37, 1-4); tolta l'armatura, $i$ chirurghi cercano invano la ferita; il Conte chiede che sia esaminata la sopravveste "insanguinata", la quale continua a essere bianca come prima, e non presenta alcun segno simile a sangue, "eccetto un nastro o una fetuccia rossa / challacciava da collo, e sciolta séra / e pendea giu per fino a la cintura" $(43,8 ; 44,1-2)$. Un nastro rosso che decorava lo scollo della sopravveste si è slacciato, e viene scambiato per una striscia di sangue: il motivo di tanto equivoco sta in un piccolo dettaglio dell'abito. Sembra una parodia; e difatti lo è. ${ }^{21}$ Nell'Orlando furioso, XXIV 64-65, Zerbino viene colpito da Durindana impugnata da Mandricardo: la ferita è lunga ma poco profonda, perché la spada ha inciso in modo superficiale la pelle, riparata dalla spessa armatura. Scorre fino a terra una sottile, rossa riga di sangue; e l'Ariosto, trascorrendo all'istante in un pacifico e lieto universo domestico (non senza una colta reminiscenza omerica), rievoca il lavoro di cucito della sua donna: "Così talora un bel purpureo nastro / ho veduto partir tela d'argento / da quella bianca man più chalabastro, I da cui partire il cor spesso mi sento" $(66,1-4)$.

Non conviene però pensare che si tratti, da parte del Tassoni, di uno stratagemma facile: un semplice gioco di prestigio di figure retoriche scambiate. Consideriamo le componenti topiche della scena. La situazione del ferito che contempla il proprio sangue uscire (nei due versi già citati della Secchia: "Caduta la visiera il Conte mira, I e vede rosseggiar la sopravesta") ammette molti riscontri, elaborati su diversi registri: anche a non dire del tragico di lacopo del Cassero "lì vid'io / de le mie vene farsi in terra laco» $e$ di Bonconte "arriva io forato ne la gola, I fuggendo a piede $e$ sanguinando il piano" (nel canto dantesco dell'acqua e del sangue, Purg. $V$ 
83-84 e 98-99), c’è l'elegiaco dell'ultima percezione di Cloridano «Del proprio sangue rosseggiar la sabbia / fra tante spade, e al fin venir si mira» (Orlando furioso XIX 15, 5-6, assai vicino alla lettera della Secchia); o il tono medio della reazione di Raimondo (la ferita è lieve, di quelle di tutti $i$ giorni) "Da l'usbergo lo stral si tragge il conte / ed ispicciarne fuori il sangue vede; / e con parlar pien di minaccie ed onte / rimprovera al pagan la rotta fede» (Gerusalemme liberata VII 103, 1-4). L'Ariosto offre anche una gradazione comica del motivo: "Gradasso disperato, che si vede / del proprio sangue tutto molle e brutto, / e ch'Orlando del suo dal capo al piede / sta dopo tanti colpi ancora asciutto" (Orlando furioso XLI 95, 1-4). L'altro elemento chiave, che in Ariosto e Tasso ha sempre un grave peso, è l'indizio della sopravveste insanguinata. Nel canto VII della Liberata, le voci sulla morte di Rinaldo si diffondono irradiandosi da un insieme concreto di oggetti, interpretati come segno certo di sciagura: le sue armi, ritrovate "rotte $e$ sanguigne", e la sopravveste, ugualmente "rotta (...) e sanguinosa» (48-49). Ma il presagio di morte più sicuro e veritiero è legato alla sopravveste di Brandimarte, nel Furioso. Fiordiligi, che l'aveva cucita e ricamata con le sue mani, la sogna diversa da quella che era: decorata da un sinistro ricamo a fitte gocce rosse; e non sa spiegarsi di aver fatto un così strano lavoro, e si spaventa (XLIII 155-56). A ragione, perché le gocce rosse, passate nel sogno, non sono che schizzi del gran fume di sangue uscito dalla ferita alla testa di Brandimarte abbattuto: "Brandimarte con faccia sbigottita / giù del destrier si riversciò di botto; / e fuor del capo fé con larga vena / correr di sangue un fiume in su l'arena" (XLI 101, 5-8). Nei funerali di Brandimarte, i colori delle vesti ubbidiscono a una rigorosa disciplina simbolica: il corteo dei frati bigi, bianchi e neri; conti e cavalieri, povera gente e paggi, tutti in "abito di duolo", con "panni negri e lunghi sin a terra", tutti "chiusi, / più che vestiti (...) di nere vestin, i cavalli con gualdrappe nere che radono il suolo. Soltanto a Brandimarte spetta il rosso: un telo di "purpurea seta" a coprire la bara, $e$ dentro, il corpo del cavaliere rivestito di un abito di porpora (XLIII 175-79). Comprendiamo infine che c'è sapienza, nell'invenzione della Secchia, e una sorta di giusto contrappasso. Il vile Conte di Culagna non ba diritto alla sopravveste vermiglia (di sangue, o di simbolica porpora), travestimento finale dei prodi, ma solo a un'esile lista di rosso ingannevole, di sangue finto.

Il vestito dello stile

Il Tassoni definisce piu volte la sua operazione innovatrice come mescolanza o mistura: l'avvertimento A chi legge dell'edizione 1630 parla 


\section{Silvia LONGHI}

della "curiosità" dell'autore "di vedere come riuscivano questi due stili mischiati insieme, grave e burlesco; imaginando che se ambidue dilettavano separati, avrebbono eziandio dilettato congiunti e misti: se la mistura fosse stata temperata con artificio" (p. 5). In una Nota introduttiva alle Dichiarazioni del Salviani, la Secchia è descritta con una

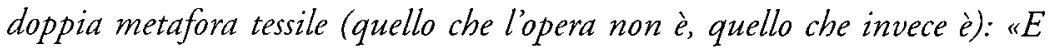
non è panno (come disse colui) tessuto a vergato, o, come disse un altro, una livrea da svizzero: ma ̀̀ un drappo cangiante, in cui mirabilmente risplendono ambidue $i$ colori del burlesco e del grave»."2 Il vergato, o vergatino, è un tessuto a righe sottili, di colore diverso. La Secchia non è fatta di tal stoffa. Il costume da guardia svizzera pontificia, a larghe strisce verticali, alternatamente chiare (gialle) e scure (blu; e alcune rosse nelle maniche), è un termine di paragone altrettanto inappropriato, anche alla luce delle Dichiarazioni: "E questo sia detto per rispondere a chi oppose già al poeta che questo era un miscere sacra profanis, e che questo poema era una calza d'uno svizzero di due assisem. ${ }^{23}$ Niente livrea da svizzero, dunque, con la sua netta partizione dei due colori. Bensì un "drappo cangiante»: una stoffa cioè iridescente, in cui la tessitura di fili di colori diversi è così serrata e compatta che il colore dell'insieme cambia continuamente, a seconda della luce, della distanza, dellinclinazione. Il cangiante è un panno che ha la virtù di trasformarsi sotto gli occhi dell'osservatore. Cosi la Secchia; cosi tutto quanto all'interno della Secchia, cavalieri e cavalli e donne e oggetti. Nel poema, se ho visto bene, questo tessuto specialissimo è nominato una volta: nel ritratto di un "cortegian fatto soldato" di grande eleganza: "L'armatura dorata $e$ rilucente / con sopraveste avea cangiante e varia" (V 33, 1-2). Possiamo aggiungere che ne fa uso il Folengo, nella Moscheis. Sui cavalli-cetonie i servi pongono coperte iridescenti di cangiante: "Imponunt famuli varias canzante copertas" (I 161); le mosche a cavallo dei grilli mandano riflessi multicolori, alcune vestite di velluto, altre di cangiante: "Stant longo moschae frenatis ordine grillis, I quarum pars maior multicolora nitet: I sunt quae velluto, sunt quae canzante teguntur" (III 33-35).24

Stoffe $e$ abiti sono termini di paragone consueti per indicare lo stile nell'ambito della letteratura giocosa. ${ }^{25}$ Comincia il Berni, ${ }^{26}$ nel secondo capitolo in lode della peste: "Cantai di lei, come tu sai, l'altr'anno, / E, com'ho detto, le tagliai la vesta / Larga, e pur mi rimase in man del panno" (XXII 10-12); o se si vuole, procedendo per negazione, nel capitolo a Ippolito de' Medici in lode del nano Gradasso: "Non sono $i$ versi a guisa di farsetti, / Che si fanno a misura, né la prosa, / Secondo le persone or larghi or strettì (XXIV 16-18; nello stesso c'è un riferimento 
aperto alla Batracomiomachia, 52-54). In quella summa di sapere stilistico che è il Ragionamento di Nicola Villani, il principio del comico viene individuato nella "sconvenevolezza» reciproca tra materia $e$ locuzione, che fa ridere "non altrimenti che rider farebbe il vedere $i$ compagni d'Ulisse con anella d'oro appiccate al grugno, o una bertuccia andante in contegno con bella guarnacca di porpora". ${ }^{-}$

La metafora del vestito dello stile, nella sua realizzazione più forte, dà nome al genere stesso dei "travestimenti". Dice il Lalli nella prefazione all'Eneide travestita, per scusarsi di aver attentato alla dignità di Virgilio: "parea che quell' incomparabile autore, già vestito di oro, di poveri e ruvidi panni travestir si volesse», e tuttavia "l'opera famosa di Virgilio, come oro ravvolto negli stracci, non perderà per questo mai la sua perfettionem. ${ }^{28}$ In una meno nota lettera apologetica a firma di tal Tomaso Giglioli, stampata alla fine dell'opera, due esempi mitologici puntellano la sentenza che "lo stile (...) nella poesia è come un vestimento". Luno, che il vedere "soggetto eroico vestito di basso stile è quanto mirare Apollo in abito di pastore" (sulla scorta di Ovidio, Metamorfosi II 679-82). L'altro, che (secondo "un'altra favola di profondo sentimento": ancora le Metamorfosi, VIII 626-27) "si compiacquero una volta Giove e Mercurio travestirsi, et andare incogniti a visitare il mondo": cosi l'Eneide, mutato abito, gode ad andare per il mondo «in apparenza alquanto vile, ma in sostanza quella medesima che veramente èm. ${ }^{29}$ Questi dèi travestiti ci riconducono al nostro poema.

La secchia, e la mania degli scambi

Di citazione in citazione, nel corso di questo studio, si sarà percepito il fatto che il verbo cambiare / cangiare ricorre nella Secchia con una frequenza allarmante. ${ }^{30}$ Al centro dell'universo instabile del poema, l'oggetto-secchia produce un vortice di scambi e baratti, perché "equivale" a un sacco di cose. Nell'esordio, sta al posto di Elena di Troia, quale movente della guerra: "vedrai, s'al cantar mio porgi l'orecchia, / Elena trasformarsi in una secchia" (I 2, 7-8), famoso proclama che dichiara per figura la mutazione imposta all'epopea. ${ }^{31}$ Ma è solo l'inizio. I Bolognesi offrono la terra di Crevalcore (paludosa e piena di rane), in cambio della restituzione della secchia: "Mentre vi soffre la fortuna in questo / di cambiare una secchia in una terra" (II 19, 1-2); per un po' sembra che il baratto possa funzionare: "Il dì che venne, il cambio fu approvato" $(21,1)$, poi l'accordo naufraga. Più avanti, gli ambasciatori bolognesi ritentano di «racquistar 
la secchia", proponendo uno scambio con i baroni presi prigionieri: "né trattar più de la proposta vecchia, I ma di cambiar la secchia in que' baroni, I eccetto il Re, ch'essi tenean prigioni» (VIII 42, 2 e 6-8). Replica il Potta che è miglior partito "finir tutte le liti e le contese, / e barattar la secchia col Re sardo" (43, 3-4); ma sulla sproporzione dello scambio col re Enzo la trattativa si arena. Il testo suggerisce anche altre equivalenze. La secchia viene usata, in modo abbastanza normale, come recipiente per bere acqua dal pozzo (I 44); ma subito dopo è adoperata come scudo: "E Spinamonte, che la secchia presa / per bere avea, spargendo l'acqua in terra / e tagliando la fune ond'era appesa, I se ne servi contro i nemici in guerra, / con la sinistra man la tien sospesa / per riparo, e con l'altra il brando afferra" (I 46, 1-6). Ci sono anche analogie più qualificate sul piano mitologico. La secchia funziona come il vaso di Pandora: "La Fama in tanto al ciel battendo l'ali / con gli avisi d'Italia arrivò in corte, / ed al Re Giove fe' sapere i mali / che d'una secchia era per trar la sorte") (II 28, 1-4). Una nota del Salviani la descrive nella sua realtà fisica, lignea e ferrea, e immediatamente la trasfigura, equiparandola alla nave Argo e all'Arca di Noè: "La secchia, che tuttavia si conserva in Modana, è veramente d'abete, e mostra che fosse nuova con tre cerchi e il manico di ferro. $\dot{E}$ anticaglia degna d'esser veduta, come quella che tiene il terzo luogo dopo la nave d'Argo e l'Arca di Noèm. ${ }^{32}$ Queste equivalenze si reggono sulla forma comune di recipiente concavo. In base proprio alla forma, e alle prime due lettere del nome, si può forse sospettare che la "secchia" sia anche un sostituto parodico del "gran sepolcro" dell'esordio della Liberata: "Canto l'armi pietose e 'l capitano / che 'l gran sepolcro liberò di Cristo". ${ }^{33}$

Tra la prima e l'ultima ottava del canto I si compie per la secchia uno straordinario spostamento, dal basso verso l'alto. In partenza è «un'infelice e vil secchia di legno" $(1,3)$, in dotazione a un pozzo appena dentro la porta San Felice a Bologna: e i nemici assetati la calano con la carrucola giù, sul fondo, fino all'acqua "ch'era assai cupa e distante" (44, 3). Da questo massimo sprofondamento, la secchia comincia la sua risalita: è bicchiere, poi scudo, infine trofeo nelle mani di Manfredi: "né potendo mostrar piu degne spoglie, / in atto di trofeo leva sublime / sopra una lancia l'acquistata secchia" (50, 5-7). Una ripetizione di tipo epico contrassegna le fasi dell'elevazione della secchia: l'arrivo trionfale a Modena: "portar la secchia in alto egli facea / da Spinamonte innanzi a la bandiera, I e di mirto e di fior cinta l'avea / si che spoglia parea pomposa e altera» (59, 3-6); e la santificazione, a guisa di preziosa reliquia $(63,1-8)$ : 


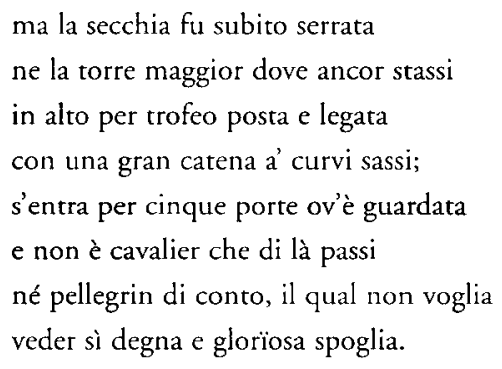

Ma come è fatta la secchia? Chi volesse vederla nella sua rustica concretezza, ne trova una fotografia nell'edizione UTET del 1928, e poi nel volumetto di Atti di un convegno tenuto nel 1990 a Villa Cesi, a Nonantola ${ }^{34} \mathrm{Ha}$ un'aria un po'sgangherata (direbbe il Berni, esagerando al suo solito: «Una barcaccia par vecchia dismessa, / Scassinata e scommessa"). Ci interessa anche vederla protagonista, nel tempo, del ciclo figurativo del libro. Una scena quasi d'obbligo è l'arrivo trionfale della secchia a Modena: è la prima illustrazione in assoluto, e anche l'unica offerta da una sola delle stampe secentesche, quella di Ronciglione (Roma, G. B. Brugiotti) del 1624. L'acquaforte dell'antiporta mostra la secchia appesa in cima alla lancia del cavaliere Spinamonte, adorna di due graziose nappine che ricadono ai lati. ${ }^{35}$ Aereo feticcio, la secchia sarà poi sempre sospesa in fragile equilibrio in cima a quella lancia: coronata di volta in volta di ghirlande di fronde, di alloro, e soprattutto di fiori. Le spetteranno anche diversi trionfi allegorici: trasportata in volo da putti alati, sopra personaggi rivestiti del costume adatto a significare la Poesia epica e la Poesia comica, oppure la Poesia e la Storia, in meravigliosa alleanza. Nella sontuosa edizione Soliani maior del 1744, la figurina stilizzata della secchia è impiegata anche per l'ornamentazione minuta. ${ }^{36}$ Il frontespizio la rappresenta in una calcografia posta sotto i titoli: in un paesaggio di campagna, con Modena sullo sfondo, la secchia giace rovesciata per terra accanto a un pozzo, e una grossa civetta con le ali ancora aperte le si è appena posata sopra. La civetta, sacra a Pallade, vorrà indicare la sapienza di Minerva che tocca una materia umile: un emblema di gusto erasmiano. Ma la vignetta più irresistibile è il finalino xilografico che si incontra, quasi per caso, dopo la Prefazione del Barotti (p. LIII): si vede la secchia ritta in dignità araldica, con le trivelle modenesi e festoni vegetali ai lati, e sormontata da un cartiglio con il motto "Avia Pervia". Appollaiata sull'orlo c'è una civetta molto piccola, sbigottita che pare un allocco, con le ali spalancate e tutte le pennine dritte in testa.

Silvia Longhi 


\section{SILVIA LONGHI}

1. Mi servo dell'edizione: Alessandro TASSONi, La secchia rapita, edizione cririca a cura di O. Besomi, Padova, 1990, voll. 2, II, Redazione definitiva, di qui cito anche, all'occorrenza, le Dichiarazioni dell'autore, scritte in persona di Gaspare Salviani, e passi delle prefazioni e degli avvertimenti, sempre del Tassoni, che hanno accompagnato la vicenda compositiva dell'opera.

2. Ibid., Dichiarazioni, p. 397.

3. Vedi vl 17,7-8 "credendolo schiacciar come un ranocchio, / d'un rovescio lcvò l'uno c l'altr' occhio"; XI 48, 8 "parve un topo caduto in mezzo a l'oglion; XI 61, 7-8 "che d'una rana imbelle c senza morso / l'avesse al fin mutato in tigre, in orsom.

4. Testo con traduzione a fronte, e ottime note introduttive e di commento, in: [OMERO], La battaglia delle rane e dei topi. Batrachomyomachia, a cura di M. Fusillo, Prefazione di F. Montanari, Appendice di C. Carpinato, Milano, 1988.

5. Uso per comodità l'edizioncina dei "Tascabili Economici Newton" (con testo greco a fronte): LUCIANO di SAmOSATA, Storia vera, prefazione di G. Toti, cura e versione di U. Montanari, Roma, 1994.

6. Teorilo Folengo, Macaronee minori. Zanitonella, Moscheide, Epigrammi, a cura di M. Zaggia, Torino, 1987, p. 408: il passo appartiene alla redazione definitiva, ma è solo lievemente variato rispetto alla Moschaea toscolanense (11 87-92, p. 341). La bella introduzione dello Zaggia al poemetto zooepico del Folengo rappresenta anche un capitolo nella storia della fortuna della Batracomiomachia.

7. Vedi i contributi di G. Petrocchi, Guerriere a cavallo, in Saggi sul Rinascimento italiano, Fircnze, 1990, pp. 117-20; c di R. Alhaique Pettinclli, Figure femminili nella tradizione cavalleresca tra Quattro e Cinquecento, in "Italianistica", anno XXI, n. 2-3 (maggio-dicembre 1992), pp. 727-38 (è il primo di due numeri che la rivista dedica alla memoria di Giorgio Varanini).

8. MatTeo Maria Bolardo, Orlando imnamonato, a cura di R. Bruscagli, voll. 2, Torino, 1995.

9. Ludovico Ariosto, Orlando Furioso, a cura di C. Segre, Milano, 1982".

10. G. Ferroni, Da Bradamante a Ricciardetto. Interferenze testuali e scambi di sesso, nel volume miscellaneo La parola ritrovata. Fonti e analisi letteraria, a cura di C. Di Girolamo e I. Paccagnella, Palermo, 1982, pp. 137-59.

11. Si cita da: Tutte le Poesie di Torquato Tasso, a cura di L. Caretti, I, Gerusalemme liberata, Milano, 1957.

12. G. Gorni, Il chiasmo di Clorinda, in "Colloquium Helveticum. Quaderni svizzeri di letterarura generale e comparatan, 2 (1985), pp. 81-95.

13. Due opere: P. Puliatti, Iconografia Tassoniana. La fortuna grafica della "Secchia rapita", Modena-Milano, 1965; e Id., Bibliografia di Alessandro Tassoni, voll. 2, I, Edizioni, II, Iconografia e critica, Firenze, 1969 e 1970.

14. O. Besomi, Un mito rovesciato, Lucrezia: un 'racconto secondo' della "Secchia rapita", in Forme e vicende. Per Giovanni Pozzi, a cura di O. Bcsomi, G. Gianella, A. Martini, G. Pedrojetta, Padova, 1988, pp. 357-82. 
15. Ho visto l'esemplare della Biblioteca Universitaria di Pavia, Corr. 93. E. 14 ( p. 334). Questa illustrazione è riprodotta in Puliatri, Fortuna grafica, cit., tavola XI; mentre è descritta, ma non riprodotta, in Id., Bibliografin II, cit., n. 487.

16. Uso la nuova edizione riccamente commentata: Francesco Petrarca, Trionfi, Rime estravaganti, Codice degli abbozzi, a cura di V. Pacca e L. Paolino, Introduzione di M. Santagata, Milano, 1996.

17. Spiega il Salviani, Dichiatazioni cit., p. 391: «A Modana si fanno e s'adoprano le maschere più che in citrà del mondo".

18. Vedi Al.essandro TASsoni, Opere, a cura di L. Fassò, Milano-Roma, 1942, p. 1062; e Id., La secchia rapita. Rime e prose scelte, a cura di G. Ziccardi, Torino, 1968;, p. 118.

19. L'imponente studio La storia nella "Secchia rapita", Modena, I Parte 1906, I1 Parte 1909, estratte dalle "Memorie della R. Accademia di Scienze, Lettere ed Arti in Modena", scrie III, voll. VI e IX, è tuttora riconosciuto come "un punto di riferimento imprescindibile e sicuro" dal Besomi, che insiste con ampie delucidazioni sulla componente storica del poema: vedi Id., Glosse d'autore e glosse d'editore: per un commento alla "Secchia rapita", in Il commento ai testi. Atti del Seminario di Ascona, 2-9 ottobre 1989, a cura di O. Besomi e C. Caruso, Basel - Boston - Berlin, 1992, pp. 373-407 (in particolare le pp. 374-79).

20. Viene in mente l'esordio del De ludo scacchorum di GIROLAMO VIDA: «Ludimus effigiem belli, simulataque veris / proelia, buxo acies fictas et ludicra regna: / ur gemini inter se reges albusque nigerque / pro laude oppositi certent bicoloribus armis" (1-4): si cita dall'edizione di Roma, Ludovico Vicentino, 1527.

21. La sensazione di déjà $v u$, persistente, avrebbe stentato a concretarsi in un risultato senza l'aiuto di uno strumento utilissimo: alludo all'ASIM, cioè all'Archivio delle Similitudini. I. Ariosto. Boiardo. Marino. Pulci. Bernardo Tasso. Torquato Tasso. Tassoni. Trissino, a cura di O. Besomi e N. Casella, Hildesheim-Zürich-New York, 1994, p. 308 (alla voce nastro) e p. 357 (alla voce piaga), che registra dalla doppia prospetriva del comparante e del comparato la similitudine ariostesca. Non è registrato invece il passo del Tassoni, dove la similitudine esiste nella sostanza, ma non è formalmente articolata: «né san cosa trovar di che segnata / sia, né ch’a sangue assomigliar si possa, I eccetro un nastro o una fecuccia rossan (xI 43, 6-8).

22. Dichiarazioni cit., p. 447.

23. Ibid., p. 410.

24. Edizione Zaggia, cir., pp. 396 e 444.

25. Qualche indicazione in proposito ho già dato all'interno di un capitoletto dedicato alle Metamorfosi dello stile piacevole tra Seicento e primo Settecento, nel Manuale di letteratura italiana. Storia per generi e problemi, a cura di F. Brioschi e C. Di Girolamo, vol. II, Dal Cinquecento alla metà del Settecento, Torino, 1994, alle pp. 307-14. Nello stesso volume, pp. 399-404, va segnalata una buona presentazione della Secchia di A. Martini.

26. Ciro dalla mia edizione delle Rime, in corso di stampa nei Poeti del Cinquecento Ricciardi.

27. [NICOLA VILlan]], Ragionamento dello academico Aldeano sopra la poesia giocosa de' Greci, de' Latini, e de' Toscani, con alcune poesie piacevoli del medesimo autore, Venezia, Giovan Pictro Pinclli, 1634, p. 47. 


\section{SilviA LONGHI}

28. Cito dalla prima edizione: L'Encide travestita del Signor Gio. Battista Lalli, Roma, s. t., 1634, c. 3 v e c. $5 \mathrm{r}$.

29. I frammenti della lettera del Giglioli dalla stessa edizione, p. 594.

30. È il verbo tecnico dei travestimenti: Venere, per ingannare Manfredi, "cangia volto, e 'l bel sembiante espresso / de la Contessa di Caserta prende»; poi, fatto quel che doveva, si ritrasforma: "subito si ritira e cangia aspetto. / Ne la forma immortal sua prima riede" (x 27, 3-4 e 37, 4-5). Bacco appare al Potta con le fattezze di "un gigante orribile e cornuto": "Bacco era questi a generar spavento / in quella forma orribile cangiato / che combattuto avea col dio di Cintom (VI 71, 3 c $73,5-7)$.

31. Vedi anche 1 48,1-4 "Non fu rapita mai con più fatica / Elena bella al tempo di Sadocco, / né combattuta Aristoclèa pudica / al par di quella secchia da un baioccon.

\section{Dichiarazioni cit., p. 387.}

33. Si registrano alcuni indizi a favore di unantipatia del Tassoni per la prima ottava della Liberata: il "Vorrei cantar" della Secchia corregge il presuntuoso "Canton in prima posizione; lo stilema «il gran sepolcro" esiste nclla storia redazionale della Secchia come variante a vIII 24,8 (vedi l'apparato); esistono anche le uarmi pietose», che sono sconfittc in una comparazione ironica, a XII 11, 5-6 «taciute avria quell'armi suc pietose / il Tasso".

34. La Secchia rapita. Atti del Convegno. Modena, Villa Cesi, 22 settembre 1990, Modena, 1991, p. 87: la sccchia cra esposta nella mostra che affiancava il convegno.

35. Ho visto l'esemplare della Biblioteca Universitaria di Pavia (Rari. A. 21), in pergamena, in cui l'acquaforce e le altre piccole incisioni (fregi, grottesche, letterine iniziali dei canti) sono state dipinte a vari colori, con tocchi dorati. L'illustrazione è riprodotta in Puliatti, Fortuna grafica, cit., tavola I, e Id., Bibliografia cit., II, n. 435.

36. Esemplare della Biblioteca Universitaria di Pavia, 66. T. 17. Nelle due edizioni Soliani del 1744 le illustrazioni a pagina intera che precedono ogni canto sono diverse (anche per tecnica: nell'editio maior acqueforti, nella minor xilografie), mentre alcuni elementi dell'ornamentazione sono identici (certe lettere iniziali, e certi finalini con la secchia retta da un putto, o con la Ghirlandina). I due frontespizi sono molto simili (riprodotti in Puliatti, Bibliografia cir.). 